\title{
Acquisition of consistent associative responses with stimulus selection disallowed
}

JOHN C. ABRA

UNIVERSITY OF CALGARY, ALBERTA

Ss who produced their own responses to stimuli (MGR) were compared with paired-associate learning (PAL) Ss in learning to a criterion of two successive consistent trials. Learning was much slower in MGR than PAL, mainly because of slower acquisition of criterion responses in MGR. The two conditions performed at a comparable level on trials following the first criterion anticipation. The two conditions did not differ in rate of forgetting despite more potential proactive inhibition in the MGR conditions.

In a rarely-used learning method known as the method of generated responses (MGR), $S$ is shown a series of stimuli in the usual paired-associate learning (PAL) manner. However, he is required to produce his own responses; learning is continued until he is able to pair a response of his own choosing consistently with a particular stimulus. A previous study (Abra, 1966) using low meaningful trigrams showed that Ss in MGR reached criterion as rapidly as a control group that learned by conventional PAL the lists generated in MGR. However, the first emission (regardless of pairing) of these criterion responses occurred earlier in PAL than MGR, suggesting that the response-acquisition stage of learning (Underwood \& Schulz, 1960) was retarded in MGR. Nevertheless, once these responses were produced and paired with their criterion stimuli, succeeding performance was appreciably better in MGR.

In contrast to Abra's results, Mattocks (described by Underwood \& Schulz, 1960, pp. 273-278), using identical stimulus materials, found criterion was reached much more slowly in MGR than in PAL. An obvious procedural difference between the two studies was that Abra allowed his MGR Ss to use the letters in a stimulus as the first letter of the corresponding response; Mattocks did not allow his Ss to use any of the stimulus letters in this manner. The purpose of the present study, therefore, was threefold; first, to repeat comparable conditions from the earlier study using Mattocks' procedure, to determine whether his results are reliable. Assuming they are, it is of more interest to know whether the greater difficulty in MGR is due mainly to greater difficulty in the response-learning stage. The earlier study suggested that most of the difficulty in MGR lay in this stage. Third, Abra (1966) found that with MGR, Ss produced many drop-out errors, i.e., responses produced during acquisition but not present in the final criterion list of responses. Contemporary interference theory (Underwood \& Postman, 1960) would predict appreciable proactive inhibition from these drop-out errors and therefore a greater rate of forgetting for MGR than for PAL. In fact, only minor differences in forgetting rate appeared between the two learning methods. The present study will again examine retention for MGR and PAL; perhaps the procedural difference will reveal the predicted difference in forgetting rate.

Subjects and materials.

Eighteen undergraduate students at Northwestern University served in the MGR condition and a like number in PAL. For all Ss, the stimuli in the list were eight trigrams having very low associative connection between adjacent letters (Underwood \& Schulz, 1960). Since only one letter occurred more than once in the list, formal similarity among stimuli was minimal. Procedure

The list was presented in four different orders at a 2:2-sec. rate, with a 4-sec. intertrial interval. In MGR, $\mathrm{S}$ spelled any three-letter word he wished during the anticipation interval, except that numbers and proper names were not allowed, nor could the response begin with any of the letters in the corresponding stimulus. Learning was to a criterion of two successive trials on which the same response was produced to each stimulus. In the PAL condition, each $S$ learned the criterion list generated by a corresponding $S$ in MGR. Thus for each $S$ in MGR there was a control $S$ who learned the same list and to the same criterion, but who saw the correct response following the anticipation interval. A control $S$ was assigned to a particular MGR list by his order of appearance for the experiment.

Recall and relearning was measured after a 24-hr. interval. Both groups were instructed to try to produce the last response given to each stimulus, starting on the first trial. Relearning was again to two successive consistent trials.

Results and Discussion

Original Learning. The mean number of trials required to reach criterion was 13.7 and 19.8 for PAL and MGR respectively, $F=7.1, p<.025$. Therefore this study replicated the Mattocks finding that when Ss are denied use of the stimulus letters, MGR learning is slower than PAL. However MGR learning was still more rapid here than the mean of $\mathbf{2 7 . 5}$ trials Mattocks' Ss required to reach an identical criterion, a discrepancy probably attributable to different $S$ populations employed in the two studies.

of central interest is the relative time required for the two stages of learning, i.e., response learning and $S-R$ association. To measure speed of response 


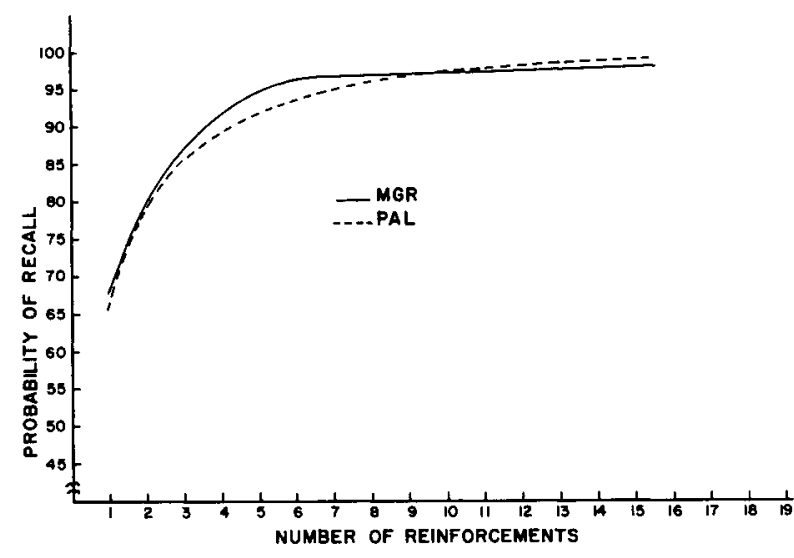

Fig. 1. Probability of a correct anticipation on the trial following each correct anticipation.

learning, the trial of first emission was noted for each criterion response in an S's protocol and a mean response-learning score computed for each $\mathrm{S}$. The mean number of trials for response learning was 3.24 and 7.34 for PAL and MGR respectively. Analysis of variance following logarithmic transformation showed this difference to be significant at the .001 level $(F=25.65)$. To measure the second or associative stage, for each criterion response the number of trials to first pairing with its criterion stimulus was determined and a mean score computed for each S. Since this mean also contained time required for response learning, the Ss response-learning mean was subtracted from this value to reveal the time required for association, once the response was available. The mean number of trials for association was .47 and 1.47 for PAL and MGR respectively. Analysis of variance of $\log (X+1)$ of these scores showed this difference to be significant $(F=7.02, p<.025)$.

Thus this study differs from the previous results (Abra, 1966) in that MGR is slower than PAL not only in speed of response learning, but also in associative learning. However, in terms of absolute differences between the two conditions, the response-learning effect is much larger, which suggests, in line with the earlier reasoning, that the overall slower learning in MGR is due mainly to increased difficulty in stage 1. However, after a criterion response is paired correctly once with its stimulus, a different pattern emerges. A multiple-entry probability analysis (Underwood, 1964) reveals for each condition the probability of another correct anticipation on the trial following each correct anticipation. The results of this analysis are shown in Fig. 1. It is clear that the two conditions differ but little in performance once criterion pairings are achieved. Abra (1966) found that the probabilities were uniformly higher in MGR when learning speeds were comparable; here, with learning speeds slower in MGR, the probabilities are almost identical.

As in the earlier study, many drop-out errors were produced in MGR. For each $S$, the total number of these errors produced was determined and this value converted to a percentage of errors per opportunity (the ratio of drop-out errors to the total number of errors committed). Analysis of variance following arcsin transformation showed that more dropout errors were committed in MGR than PAL, F= $19.0, p<.01$. As pointed out earlier, these errors should produce substantial proactive inhibition at recall in MGR, since interference theory would expect these errors to be extinguished during learning but recover over time and interfere at recall. Mean recall was 6.61 and 6.33 for PAL and MGR respectively, a difference entirely attributable to chance $(F<1)$. Similarly, the relearning scores did not differ. Apparently here, as in the previous study, the drop-out errors produced little proactive inhibition at recall in MGR.

Usually, when retention is to be compared for conditions differing in speed and therefore degree of learning, a probability analysis is necessary to predict immediate recall for each condition, so that rates of loss may be compared (Underwood, 1964). Despite the different rates of learning here, the use of loss scores does not seem necessary. The probability curves shown in Fig. 1, from which immediate recall would be predicted for each condition, are so similar as to make it highly unlikely that predicted immediate recall would differ between the two conditions. The almost identical $24-\mathrm{hr}$. recall scores are sufficient to show that the two conditions have comparable rates of forgetting.

A general point needs to be made in regard to probability analyses. Abra (1966) showed differing probabilities despite identical learning rates; this study showed comparable probabilities despite different learning rates. Obviously, when retention is to be studied for two conditions treated differently during learning, comparable speeds in reaching criterion do not allow a certain conclusion that final degrees of learning are comparable. In such cases, a determination of probability curves should be de rigueur.

\section{References}

Abra, J. C. Acquisition and retention of consistent associative responses with varied meaningfulness and similarity of stimuli. Unpublished doctoral dissertation, Northwestern University, 1966.

Underwood, B. J. Degree of learning and the measurement of forgetting. J. verbal L\&arn. verbal Behav., 1964, 3, 112-129.

Underwood, B. J., \& Postman, L. Extraexperimental sources of interference in forgetting. Psychol. Rev., 1960, 67, 73-95.

Underwood, B. J., \& Schulz, R. W. Meaningfulness and verbal learning. Philadelphia: Lippincott, 1960. 